\title{
Bienestar animal durante el transporte y su relación con la calidad de la carne bovina
}

\section{Animal welfare during transport and its relationship with meat quality}

\author{
Marlyn Romero P, ${ }^{1 *}$ M.Sc, Jorge Sánchez V, ${ }^{1}$ M.Sc. \\ 1Universidad de Caldas, Facultad de Ciencias Agropecuarias, Programa Medicina Veterinaria y \\ Zootecnia, Departamento de Salud Animal. Manizales, Colombia. *Correspondencia: marlyn. \\ romero@ucaldas.edu.co.
}

Recibido: Marzo de 2010; Aceptado: Junio de 2011.

\section{RESUMEN}

El bienestar animal (BA) es un elemento diferenciador en la comercialización de la carne bovina a nivel internacional, aunque no forma parte de los acuerdos comerciales, se pueden citar ejemplos de las experiencias de Chile, Argentina, Brasil y Uruguay con la Unión Europea, que los ha privilegiado para la exportación de carne fresca bovina con este valor agregado. Durante el transporte, cargue y descargue los bovinos son sometidos a factores estresantes que afectan su bienestar y la calidad de la carne, además de producir importantes pérdidas económicas a los productores. Este trabajo presenta una revisión sobre el bienestar animal durante el transporte, y su relación con la calidad de la carne bovina.

Palabras clave: Bienestar animal, calidad de la carne, estrés, transporte (Fuente:CAB).

\begin{abstract}
Animal welfare is a distinguishing element within the international meat trade, although not part of trade agreements, there are examples of the experiences in Chile, Argentina, Brazil and Uruguay with the European Union that has favored the export of fresh meat with this added value. During transportation, loading and unloading, cattle is subjected to stress factors that affect their welfare and meat quality, aside from producing great economic loss to producers. This work presents a review on animal welfare during transport and its relationship to meat quality.
\end{abstract}

Key words: Animal welfare, meat quality, stress, transport (Source:CAB). 


\section{INTRODUCCION}

El bienestar animal (BA) se ha convertido en un importante atributo en el concepto de calidad sensorial y ética de los alimentos de origen animal, y un tema de interés en el comercio internacional de la carne bovina, debido a su importancia y contribución para la sanidad animal y la productividad de la ganadería, motivo por el cual la Organización Mundial de Sanidad Animal (1), ha recomendado que los servicios veterinarios de control oficial establezcan principios de bienestar en su legislación, reafirmando así la sanidad animal como un componente del BA $(2,3)$.

Adicionalmente, existe una creciente preocupación por parte de los consumidores que reclaman que los animales sean producidos en toda la cadena agroalimentaria (desde la granja hasta el beneficio) bajo estándares de bienestar aceptables, y manejados de forma humanitaria $(2,4)$. En Sur América, la implementación de prácticas de BA en las cadenas productivas cárnicas no es una prioridad generalizada, debido a situaciones socio-económicas y culturales; sin embargo, los principales países exportadores de carne: Brasil, Argentina, Uruguay y Chile, han encontrado en el BA un elemento diferenciador para la comercialización de sus productos y como una oportunidad para incluir estos aspectos en sus programas de aseguramiento de la inocuidad y en la legislación sanitaria (4).

Colombia tiene un sistema de comercialización del ganado bovino, que requiere trasladar los animales desde las zonas productoras hacia las centrales de beneficio, siendo necesario recorrer grandes distancias y afrontar diferentes condiciones geográficas, lo cual implica tiempos de transporte y de ayuno prolongado, factores que generan estrés y repercuten en la calidad de la carne $(4,5)$. Se considera que el estrés es un indicador de la pérdida de $B A$; su aparición está relacionada con el cambio del comportamiento fisiológico de algunos bioindicadores hormonales como el cortisol, y variables sanguíneas como glucosa, creatinfosfoquinasa y urea, entre otros (5-7).

El trasporte, embarque y desembarque son etapas que generan altos niveles de estrés en el bovino, provocando pérdidas económicas relacionadas con decomisos por contusiones de diferente grado, mortalidad animal, bajo rendimiento de la canal y alteración de las variables organolépticas de la carne, entre otros aspectos (8). Dada la importancia del BA y sus implicaciones en la calidad de la carne, el presente artículo pretende discutir sobre los factores desencadenantes de estrés durante el transporte, cargue, descargue, y su relación con la calidad de la carne bovina.

Estrés y bienestar animal. El concepto de BA está basado en la relación armoniosa del animal con el medio; en esta relación entran a jugar un papel importante su estado físico y psicológico (7). Se han descrito como condiciones básicas que aseguran el bienestar de los animales cinco componentes que se han denominado "las cinco libertades": 1) Libre de hambre, sed o un nivel de nutrición insuficiente; 2) No presentar dolor, heridas o enfermedad; 3 ) Libre de temor o angustia; 4) No presentar incomodidad; 5) libre de manifestar un comportamiento natural, las cuales deben regir el BA (1). La percepción de calidad de vida de los animales no sólo incluye la ausencia de sufrimiento, sino también la calidad de las relaciones de éstos con el ambiente de manera que puedan satisfacer sus necesidades preferenciales (9).

Las actividades pre-sacrificio incluyen las prácticas y condiciones aplicadas al bovino durante el período comprendido entre la movilización y el trasporte desde la granja, hasta la insensibilización (10). Durante este período los animales son sometidos a factores desencadenantes de estrés que incluyen: 1) incremento del manejo, recolección y arreo con elementos punzantes o con tábano eléctrico, 2) mezcla de animales de diferente procedencia y contacto con personal extraño, 3) transporte y desafíos físicos como rampas, superficies resbaladizas, densidad de carga, movimiento, ruido y vibración del vehículo, 4) contacto con ambientes nuevos y no familiares, 5) privación de alimento y agua; 6) cambios en la estructura social, 7) cambios en las condiciones climáticas como temperatura, radiación y humedad, 8) imposibilidad de descanso, entre otros aspectos (10-12). Estos factores desencadenan reacciones inevitables en el animal que se traducen en estrés físico, fisiológico y psicológico (13).

El primero se genera por el esfuerzo físico del animal durante el arreo, el cargue y descargue del camión, así como el intento para mantenerse en píe durante el movimiento del vehículo. El estrés fisiológico puede ser medido en términos de cambios de la homeostasis del animal, por la privación de alimento y agua, la capacidad de utilizar sus reservas en el mantenimiento de la temperatura corporal y actividad física, o para superar alguna lesión o enfermedad. El psicológico, es el percibido por la conciencia animal, siendo por lo tanto difícil de medir objetivamente $(12,14,15)$. 
El estrés ha sido utilizado como indicador de la pérdida de BA y es definido como la acción de estímulos nerviosos y emocionales provocados por el ambiente sobre los sistemas nervioso, endocrino, circulatorio y digestivo de un animal, produciendo cambios medibles en los niveles funcionales de estos sistemas, en especial altera la homeostasis interna induciendo cambios en la actividad del sistema nervioso autónomo y el eje hipotálamo-pituitaria-adrenal-HPA (7). Se ha denominado "Diestrés" cuando la repuesta del animal al factor estresante pone realmente en riesgo su bienestar (16).

De acuerdo con la duración y sus efectos el estrés puede ser agudo (transitorio) o crónico (de largo efecto)(17). En cualquier caso, una vez que el sistema nervioso central percibe una amenaza, se desarrolla una respuesta que consiste en una combinación de las cuatro respuestas generales de defensa biológica: comportamiento, sistema nervioso autónomo, inmune y neuroendocrino. A pesar de que los cuatro sistemas biológicos de defensa están disponibles para que el animal responda a un factor estresante, no todos los cuatro son necesariamente utilizados contra todos los factores de estrés. En particular, la homeostasis se mantiene cuando sólo los dos primeros mecanismos están involucrados; por el contrario, cuando los cuatro mecanismos de defensa han sido implicados, algunas de las funciones biológicas pueden verse modificadas adversamente y los animales estarán en peligro (diestrés) (17).

Dentro de la respuesta neuroendocrina tienen vital importancia los sistemas: simpáticosuprarrenal-SS y el HPA, donde la activación de cualquiera de los dos depende del factor estresante que está produciendo el estímulo $(14,18)$. En la activación del primero denominado "Síndrome de emergencia", el organismo se prepara para hacer frente a peligros súbitos generando una respuesta de carácter rápida y breve, que conlleva a la activación neuronal del hipotálamo y la liberación de adrenalina y noradrenalina desde la médula adrenal, encargadas de poner al animal en estado de alerta, preparándolo para luchar o huir, provocando un aumento de la frecuencia cardiaca, vasoconstricción periférica, hiperglicemia, midriasis, hiperventilación, aumento del volumen sanguíneo y del gasto cardiaco (17).

En el eje HPA se presenta la liberación del Factor Liberador de Corticotropina (CRH) y la vasopresina en el hipotálamo, que actúan sobre la hipófisis anterior estimulando la liberación de la Hormona Adenocorticotrópica (ACTH), la cual es liberada al torrente sanguíneo para estimular la síntesis y secreción de glucocorticoides (GC), especialmente cortisol desde la corteza adrenal. Simultáneamente se estimula la liberación de catecolaminas (adrenalina, noradrenalina y dopamina) desde la médula adrenal, así como hormonas tiroideas (17). Por su parte, el cortisol aumenta la disponibilidad de energía y las concentraciones de glucosa en la sangre, porque estimula la proteólisis, lipolisis, la gluconeogénesis en el hígado, e inhibe la liberación de insulina (Figura 1)(17). En esta compleja respuesta fisiológica se presenta un proceso de retroalimentación negativa, permitiendo que el cortisol actúe sobre el hipotálamo y la hipófisis disminuyendo la producción de CRH y ACTH. En esta etapa el organismo intenta adaptarse o afrontar la presencia de los factores que percibe

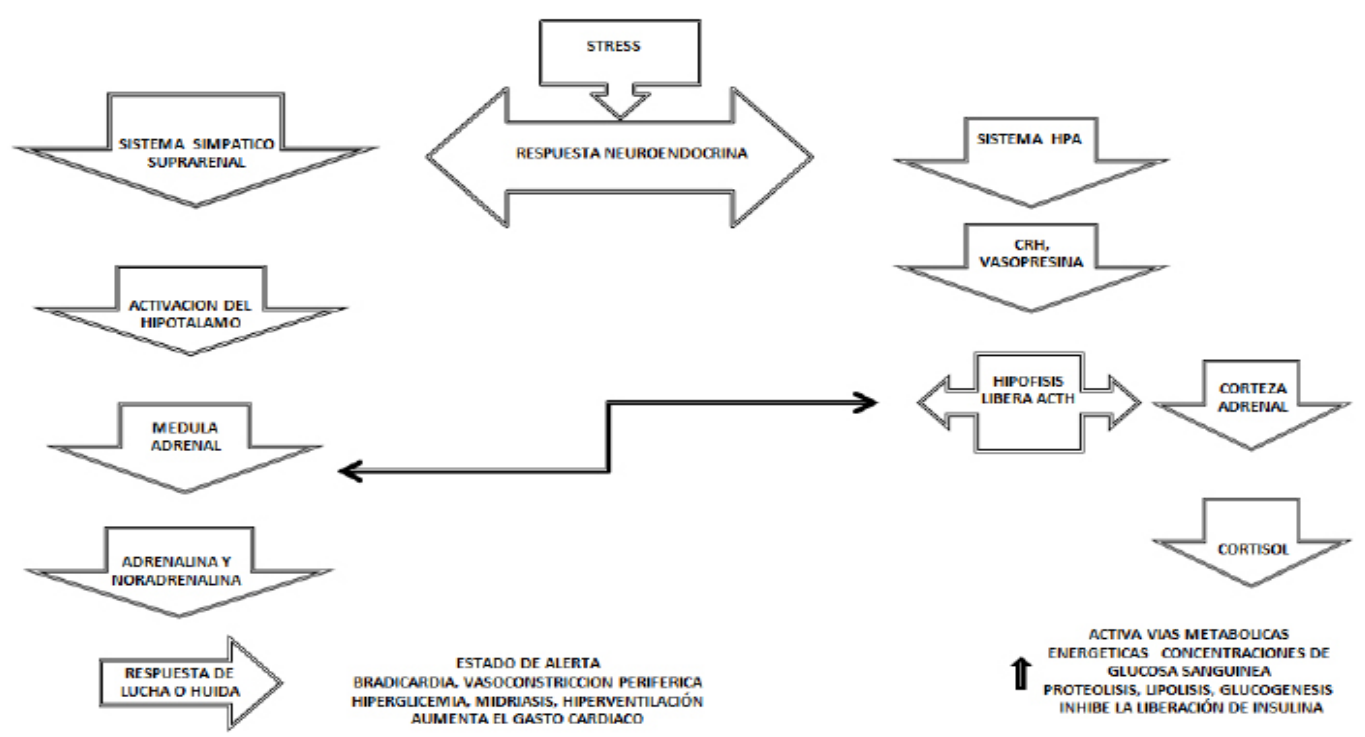

Figura 1. Esquema de los mecanismos de estrés en el animal. 
como amenaza, en donde se presenta una normalización de los niveles de GC y por ende la desaparición del estado de estrés, etapa que se ha denominado "de resistencia o relajación" (16).

El estrés crónico consiste en un estado de activación fisiológica en curso, que se presenta cuando el animal se expone a varios factores o a la exposición repetida a los mismos estresores agudos, etapa en la cual el sistema nervioso autónomo rara vez tiene la oportunidad de activar la respuesta de relajación. En este caso, se presenta una sobreexposición a las hormonas del estrés, que produce un costo biológico suficiente para alterar las funciones biológicas y producir diestrés. El estrés crónico coincide con un estado de larga duración del animal, como un problema de salud grave, que no permite su recuperación satisfactoria, en donde la intensidad y duración del sufrimiento contribuye a la severidad de la respuesta del animal. Por lo tanto, el estrés crónico es una condición de mala adaptación que puede estar asociada con una reducción directa en el nivel de BA. Por otra parte, esta condición puede afectar la susceptibilidad a las enfermedades o favorecer su progresión (17).

Aunque la respuesta al estrés es muy variable y dependiente de la capacidad de cada animal para responder, resulta evidente que si el agente estresante actúa por largo tiempo (transporte y ayuno prolongado), el efecto encontrado será mayor, sea alta o baja la capacidad de respuesta de cada animal. Por ello, mientras más largo son los tiempos de transporte y ayuno, mayores probabilidades existen de presentar estrés, afectando negativamente el BA (19).

Existe una variedad de parámetros de comportamiento, fisiológicos, bioquímicos, inmunológicos y patológicos que han sido propuestos para evaluar la capacidad de respuesta de los animales ante el estrés agudo (Tabla 1). Dentro de los biomarcadores descritos sobresalen la medición de cortisol sérico, las concentraciones de albúmina plasmática, urea, globulina, proteínas totales, la actividad de Creatin-fosfoquinasa (CK), B-Hidroxibutirato (B-OHB), actoglobina, fibrinógeno, el volumen celular empaquetado (VCP) y el conteo de leucocitos (5). Estas variables se utilizan como biomarcadores especialmente cuando se están comparando valores previos y posteriores a un determinado manejo que se cree induce estrés (19).

Bienestar animal y transporte. En Sur América el transporte de los animales desde la granja a las plantas de sacrificio se hace generalmente por vía terrestre y es efectuado
Tabla 1. Principales indicadores de estrés agudo en bovinos que permiten evaluar el Bienestar animal durante el transporte.

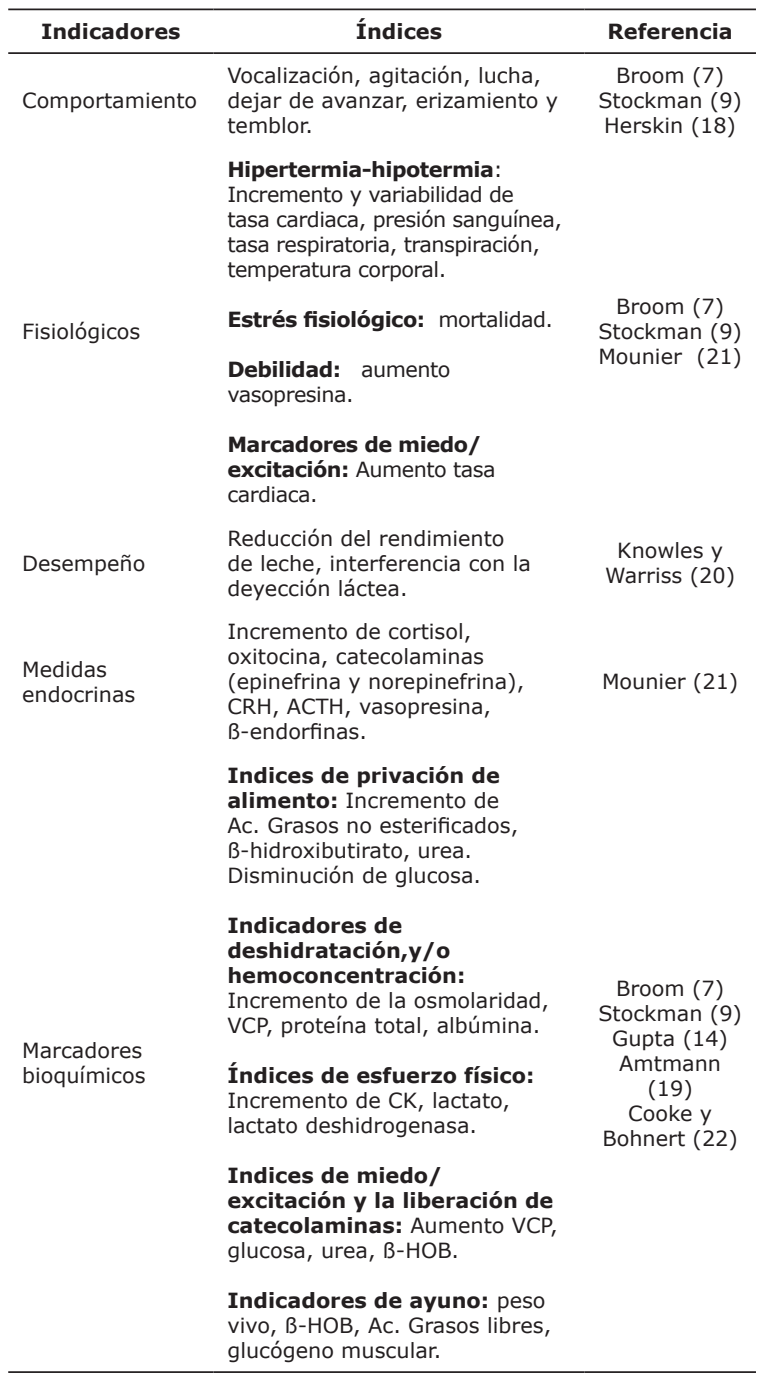

Adaptado de Knowles y Warriss (20).

por personal poco especializado o no capacitado en el transporte de bovinos $(4,12,23)$.

Estudios realizados en Chile reportan que la duración del transporte se encuentra entre 1 a $12 \mathrm{~h}$, pero ocasionalmente puede incluir hasta $60 \mathrm{~h}$. Los incrementos en el viaje se deben a las malas condiciones de mantenimiento de las vías y vehículos, características geográficas, variaciones climáticas, o a la cadena de intermediarios en la comercialización del ganado (24). El tiempo de transporte prolongado aumenta las pérdidas de peso vivo que pueden estar entre el 1.5 y $9 \%$, los riesgos de caída, muerte y contusiones de los bovinos, que se traducen en pérdidas económicas por eliminación de tejido contuso, menor rendimiento en canal y descenso en la categoría de tipificación de las canales (4). 
Los hematomas y las marcas de elementos punzantes, palos, picanas eléctricas y otros elementos de arreo inadecuados, se observan fácilmente en el proceso post-mortem, en forma de hemorragias petequiales en las canales y lesiones de distinta forma, profundidad y extensión, que son un reflejo de deficientes condiciones de manejo de los animales y pobre BA (24). Estas se han clasificado en 3 categorías: Tipo 1, cuando sólo se compromete el tejido subcutáneo; Tipo 2, tejido subcutáneo y muscular, y Tipo 3, tejido subcutáneo, muscular y óseo (25). Se ha descrito una relación directamente proporcional entre la frecuencia de presentación de las lesiones y el tiempo o distancia del transporte (19).

Una investigación realizada en África Occidental evaluó los tipos de lesiones más frecuentes en bovinos cebú comercial (Bos indicus), estableciendo que las heridas, contusiones y laceraciones localizadas a nivel de abdomen y tórax fueron las de mayor presentación, siendo menos significativas las fracturas, dislocaciones y la hernia abdominal. Estas lesiones llevaron al decomiso de tejidos superficiales y profundos que representaron en promedio entre un 1 y $3.2 \%$ del peso de la canal (12). Diferentes estudios han reportado que los altos porcentajes de lesiones de los bovinos durante el transporte están relacionados con la presencia de animales con cuernos; los golpes proferidos por los operarios con elementos contundentes; malas prácticas de conducción; mal diseño y mantenimiento de la carrocería de los vehículos, y la falta de sistemas de protección contra cambios climáticos, entre otros aspectos $(12,23,26)$. Sin embargo por las condiciones de manejo de los animales en las plantas faenadoras, otros investigadores han observado que más del $50 \%$ de las contusiones sufridas por los animales se presentan después de haber ingresado a los establecimientos (10).

Los bovinos durante el transporte pierden agua a través de la respiración, micción, heces y evaporación por termorregulación (27). En los viajes prolongados ( $\geq 18 \mathrm{~h}$ ) se hace necesaria la provisión de agua y alimento en el vehículo, así como de paradas que garanticen el descanso de los bovinos, prácticas que son poco frecuentes en los países latinoamericanos y que en Colombia están incluidas en la legislación sanitaria $(4,28)$.

La disponibilidad de espacio permitida a los bovinos en los camiones, es otro factor que incide en el bienestar animal $(7,10)$. Las altas densidades de carga, dificultan los movimientos de adaptación para mantener el equilibrio en el vehículo en desplazamiento, reportándose que a menor espacio asignado por animal es mayor la incidencia de contusiones, se incrementa la tasa cardíaca y aumentan los movimientos del animal en el vehículo, lo que favorece las caídas y lesiones de los bovinos $(8,10)$. Se ha recomendado asignar áreas que varían entre 0.7 y $1.7 \mathrm{~m}^{2}$ por animal, dependiendo de la raza, peso, localización geográfica, temperatura, entre otros aspectos $(12,27)$.

El cargue y descargue de los bovinos son actividades que pueden generar más estrés que el mismo transporte, sin embargo, no existen criterios claros que definan las condiciones apropiadas o el tiempo límite para estos procedimientos (3). Algunos estudios han sugerido que el cargue comparado con el descargue, presenta mayores efectos adversos al BA, haciendo énfasis en que la falta de capacitación del personal y el desconocimiento del comportamiento animal, serían uno de los factores que más inciden en estas etapas $(7,29)$.

Relación entre bienestar animal-estréscorte oscuro. El efecto del estrés crónico sobre la reducción de glucógeno muscular ha sido bien documentada, al igual que su asociación directa con la presentación de carne de corte "oscuro" o carne DFD (Dark, firm, dry), que se presenta especialmente en bovinos y se observa en las primeras horas después del sacrificio $(4,10)$. El estrés agota las reservas de glucógeno muscular y disminuye la formación de ácido láctico, motivo por el cual el $\mathrm{pH}$ después del sacrificio permanece alto ( $\geq 5.8$ ) (19). Un animal vivo, obtiene la energía requerida para la actividad muscular del glucógeno que se encuentra almacenado en el tejido muscular esquelético, y en mayor proporción en el hígado, en donde alcanza un valor del 2 al $10 \%$ del peso total del órgano (30).

En un animal sano y descansado, el nivel de glucógeno muscular es alto (75 - 120 mmol/Kg), pero puede descender a valores críticos (45 - 57 $\mathrm{mmol} / \mathrm{Kg}$ ), y aún así se puede alcanzar un $\mathrm{pH}$ en la canal con un valor entre 5.6 y 5.8 , el cual se considera como óptimo para el proceso de maduración de la carne (30). Cuando el animal es sacrificado, se interrumpe el suministro de sangre a los tejidos y por lo tanto de oxígeno y de nutrientes (31). El músculo intenta mantener el aporte de energía, activando las vías anaerobias que son insuficientes para cubrir la demanda energética, dando como resultado el consumo del glucógeno muscular y la posterior acumulación de acido láctico en el espacio intra y extracelular. 
La acumulación de hidrogeniones provenientes del ácido láctico es directamente responsable del descenso del $\mathrm{pH}$ y cesa hasta llegar al $\mathrm{pH}$ último ( $\mathrm{pHu}$ ), el cual se alcanza cuando se agotan las reservas de glucógeno, o se pierde la actividad enzimática por desnaturalización de las enzimas glucolíticas, proceso que ocurre en el músculo bovino entre las 18 y 36 horas postmortem $(10,31)$.

En el ganado bovino los valores bajos de glucógeno durante el pre-sacrificio se asocian a factores físicos y agentes estresantes (30), dentro de los cuales se reportan: el tiempo de transporte (32), tiempo de espera en la planta (15), alteraciones sociales en la manada (33), actividad física y fatiga, traumatismos (15), entre otros; así como a factores inherentes al individuo como género, raza, peso, y la alimentación (10). El color oscuro del músculo está relacionado con el mal sangrado de los bovinos y a la baja oxigenación de la mioglobina muscular, la sequedad se presenta por la elevada capacidad de retención de agua por parte de las proteínas sarcoplasmáticas $(19,34)$.

Estas carnes por su aspecto oscuro y consistencia dura y seca, son utilizadas para procesos industriales (elaboración de productos cárnicos), disminuyendo su valor comercial (7). En algunos países durante la inspección post-mortem, son decomisadas porque suponen un riesgo sanitario por la migración de microorganismos intestinales a las masas musculares profundas y posterior crecimiento microbiano, disminuyendo de esta forma la vida útil del producto (35). De otra parte, las carnes con elevado $\mathrm{pH}$ limitan las posibilidades de exportación y no son aptas para el empacado al vacío (19).

Bienestar y su relación con la calidad sensorial de la carne. El transporte puede producir efectos adversos en las características de la canal en variables como el $\mathrm{pH}$, color, textura y la capacidad de retención de agua (34). El pH a su vez influye en el color, la textura, el sabor, la capacidad de retención del agua y la vida útil de la carne; siendo el color de la carne una de las más importantes características que orientan la decisión de compra de los consumidores (25).

Los tiempos de transporte superiores a 16 h y tiempos de espera prolongados reducen de manera marcada la calidad de las canales de novillos, aspecto que se evidencia por un incremento de aproximadamente un $10 \%$ en la aparición de carnes clasificadas como corte oscuro $(\mathrm{pH} \geq 5.8)$ y la disminución de peso de la canal (25). El color de la carne está influenciado por el nivel de glucógeno muscular antes del sacrificio y este se reduce cuando los animales han sufrido ayuno, produciendo carnes con $\mathrm{pH}$ alto y color oscuro, característica que se ve afectada además, cuando se incrementan las interacciones sociales de los bovinos (monta, embestida, empujones, confrontaciones, entre otros) durante el transporte y la estadía en la planta $(6,25)$. Sin embargo, varias investigaciones han demostrado que transportes hasta de $6 \mathrm{~h}$ pueden afectar significativamente el $\mathrm{pH}$, la textura, la jugosidad y el color de la carne (34).

La terneza o textura de la carne es considerada como el factor más determinante de la satisfacción del consumidor (35). Esta cualidad organoléptica depende de factores biológicos como la especie animal, la edad, el sexo y el tipo de músculo; así como de factores ambientales como la nutrición, el estrés ante-mortem, las condiciones de sacrificio y refrigeración, entre otros aspectos. De otra parte, está asociada con la tasa de glucólisis, la disminución de la temperatura post-mortem y el pHu en los músculos bovinos (36). El máximo valor de dureza de la carne se encuentra en rangos de $\mathrm{pH}$ entre 5.8 y 6.3 (35). Aunque se ha reportado el aumento de la terneza en carnes con $\mathrm{pH}$ mayores a 6 , atribuibles a la actividad de las enzimas calpainas; lo mismo se reporta con pH menores, pero relacionado con la acción proteolítica del músculo bovino (36). Se ha evaluado la relación entre el tiempo de transporte y la calidad de la carne, sugiriendo una relación indirecta, es decir a mayor tiempo menor terneza; asímismo, se ha indicado que cuando las condiciones de manejo durante el transporte son adecuadas, los animales son del mismo sexo, raza y el ambiente social se mantiene estable, no se afecta la calidad instrumental de la carne, pero estas condiciones no son las que predominan en las plantas de beneficio comerciales (6).

\section{EL BIENESTAR ANIMAL EN COLOMBIA}

A partir del año 2007 se incluyó el concepto de BA en la legislación sanitaria en toda la cadena cárnica bovina. El Ministerio de Agricultura y Desarrollo Rural e Instituto Colombiano Agropecuario (ICA), lo integraron a la producción primaria mediante la Resolución 002341 de 2007 (28); y durante el transporte hacia las plantas de beneficio: Decretos 3149 de 2006 (37) y 414 de 2007 (38), y la Resolución 002341 de 2007 (28); el Ministerio de la Protección Social en el proceso de faenado en el Decreto 1500 de 2007 (39) y la Resolución 2905 de 2007 (40). 
A pesar de existir una legislación actualizada e integral, investigaciones preliminares aún no publicadas por los autores, han dilucidado que el BA especialmente durante el transporte de animales en Colombia no es una actividad especializada, el diseño de los vehículos, en lo referente a la carrocería y el piso, favorece la presencia de elementos que producen contusiones a los animales, están desprovistos de agua y de sistemas apropiados para la eliminación de excretas. En la planta de sacrificio el diseño de las instalaciones como rampas de descargue, corrales de recepción, áreas de conducción y sistemas de insensibilización no facilita el manejo y el BA. Los bovinos son sometidos a tiempos de transporte prolongado (entre 12 - $24 \mathrm{~h}$ ) y ayuno excesivo (77 h en promedio). De otra parte, el recurso humano evaluado no ha recibido capacitación específica para el transporte de animales, es generalizado el uso de elementos contundentes para el manejo de los bovinos, no se realiza una planificación del viaje y en general se desconoce el impacto del BA en la calidad e inocuidad de la carne, así como las pérdidas económicas relacionadas con éste.

Necesidades de investigacion e implementacion. La investigación debe jugar un papel crucial en la determinación de la eficiencia y la mejora de los métodos de aturdimiento, de instrumentos para evaluar el BA en condiciones de campo y en el desarrollo de nuevos métodos de insensibilización para todas las especies. Con relación a las condiciones de infraestructura, es necesario trabajar en el diseño de equipos e instalaciones para el cargue, descargue y calcetas para movilizar los animales (29). Asímismo, estudios de tamizaje en el área de Metabolómica, transcriptómica y proteómica, para evaluar proteínas y otras moléculas presentes en la célula que pueden ser utilizadas para identificar a nivel celular los efectos del estrés durante el sacrificio y predecir su incidencia sobre la calidad de la carne (41). Otras áreas de interés en donde la academia puede dar aportes importantes al conocimiento del BA se presentan en la tabla 2.

Tabla 2. Algunas necesidades de investigación en el ámbito de BA en Colombia.

\begin{tabular}{l} 
Necesidades de investigación \\
\hline Desarrollo de indicadores estandarizados que reflejen el estado \\
actual de BA en los sistemas productivos. \\
Establecer las condiciones sanitarias reales del transporte y el nivel \\
de implementación de prácticas de BA. \\
Evaluación del estrés mediante mediciones del comportamiento y \\
variables sanguíneas, su relación con la calidad e inocuidad de \\
la carne. \\
Estudios de comportamiento animal bajo condiciones comerciales de \\
manejo. \\
Identificar las principales fuentes de estrés durante el transporte y el \\
sacrificio para detectar necesidades de la industria. \\
Estudios de biología molecular para identificar biomarcadores \\
celulares de estrés.
\end{tabular}

Con relación a las estrategias requeridas para la implementación de prácticas de BA en la cadena cárnica bovina, la tabla 3 presenta algunas sugerencias que pueden ayudar a esta tarea.

Tabla 3. Estrategias para la implementación de prácticas de bienestar animal en la cadena cárnica bovina.

\begin{tabular}{l}
\hline \multicolumn{1}{c}{ Estrategias de mejoramiento } \\
\hline Reducir los manejos estresantes en la finca. \\
Implementación de tecnología, prácticas de BA y mejoramiento de la \\
infraestructura de las instalaciones y vehículos. \\
Diseño de acuerdo con el comportamiento de la especie. \\
Incentivos para los productores. \\
Diferenciación de precios. \\
Integrar el BA dentro de las políticas de inocuidad. \\
Capacitación al recurso humano operativo, técnico y profesional. \\
Certificación de transportadores de ganado a través de entrenamiento \\
o acreditación de competencias. \\
Mantenimiento de equipos de aturdimiento y uso de tecnologías que \\
garanticen el BA en esta etapa.
\end{tabular}

Siendo importante resaltar que uno de los inconvenientes más destacados para la implementación de la normatividad relacionada con el BA tiene que ver con la falta de incentivos para los productores. En la Comunidad Europea y en Estados Unidos los productos obtenidos bajo estos criterios tienen un precio de mercado diferenciado y en este último país las granjas que han adoptado estos criterios reciben subsidios por parte del Estado (42).

De otra parte, se requiere el entrenamiento y la capacitación específica del recurso humano responsable del manejo bovino en toda la cadena cárnica, que incluya el reconocimiento de incentivos, la validación de competencias y la certificación de los transportadores de ganado. Varios estudios han demostrado que la experiencia y la habilidad de los conductores reducen las lesiones del ganado durante el transporte $(23,27)$.

Es urgentela adopción de infraestructura, equipos y aplicación de lineamientos de diseño sanitario durante el presacrificio. Así como el desarrollo de investigación orientada a la resolución de problemas del medio externo, estrategia que ha demostrado en países como Canadá, Estados Unidos, Australia, Chile Uruguay y España, que es posible transferir conocimiento generado por los investigadores en el ámbito del BA e implementarlo en la producción pecuaria, lo cual ha generado posicionamiento de sus productos en el mercado, valor agregado, y el desarrollo de programas de entrenamiento y capacitación, que han permitido que el sector se fortalezca y mejore su productividad $(27,32)$. 
En conclusión el BA durante el presacrificio es un requerimiento legal en Colombia y aunque su nivel de implementación es bajo, es necesario fortalecer la investigación en la cadena cárnica bovina, capacitar al recurso humano técnico y profesional, en especial a las nuevas generaciones de profesionales relacionadas con la salud y producción animal, mejorar la infraestructura del transporte y beneficio, e integrar este componente a la política de calidad e inocuidad de la carne.

\section{REFERENCIAS}

1. OIE Organización Mundial de Sanidad Animal. Código Sanitario para los animales terrestres 2011. Título 7 Bienestar de los animales. [Consultado Noviembre 2011]. URL Disponible en: http://www.oie.int/es/ normas-internacionales/codigo-terrestre/ acceso-en-linea/.

2. Rojas $H$, Stuardo $L$, Benavides D. Políticas y prácticas de bienestar animal en los países de América: estudio preliminar. Rev sci tech Off int Epiz 2005; 24(2):549-565.

3. María GA, Villarroel M, Chacón G, Gebresenbet G. Scoring system for evaluating the stress to cattle of commercial loading and unloading. Vet Rec 2004, 26:818-821.

4. Gallo C. Using scientific evidence to inform public policy on the long distance transportation of animals in South America. Vet Ital 2008; 44(1):113-120.

5. Buckham Sporer KR, Weber PSD, Burton JL, Earley B, Crowe A. Transportation of young beef bulls alters circulating physiological parameters that may be effective biomarkers of stress. J Anim Sci 2008; 86:1325-1334.

6. Partida JA, Olleta JL, Campo MM, Sañudo C, María GA. Effect of social dominance on the meat quality of Young Friesian bulls. Meat Sci 2007; 76:266-273.

7. Broom DM. The effects of land transport on animal welfare. Rev sci tech Off int Epiz 2005; 24(2):683-691.

8. Gallo C, Tadich N. Transport of cattle for slaughter: effects on animal welfare and meat quality. Agro-Ciencia 2005; 21(2):37-49.

9. Stockman $\mathrm{CA}$, Collins $\mathrm{T}$, Barnes $\mathrm{AL}$, Miller D, Wickham SL, Beatty DT et al. Qualitative behavioural assessment and quantitative physiological measurement of cattle naïve and habituated to road transport. J Anim Prod Sci 2011; 51:240-249.
10. Ferguson DM, Warner RD. Have we underestimated the impact of pre-slaughter on meat quality in ruminants?. Meat Sci 2008; 80:12-19.

11. de Witte K. Development of the Australian Animal Welfare standards and guidelines for the land transport of livestock: Process and philosophical considerations. J Vet Behav 2009; 4:148-156.

12. Minka NS, Ayo JO. Effects of loading behaviour and road transport stress on traumatic injuries in cattle transported by road during the hot-dry season. Livest Sci 2007; 107:91-95.

13. Fisher AD, Colditz IG, Lee C, Ferguson DM. The influence of land transport on animal welfare in extensive farming systems. J Vet Behav 2009; 4:157-162.

14. Gupta S, Earley B, Crowe MA. Effect of 12hour road transportation on physiological, immunological and hematological parameters in bulls housed at different space allowances. Vet J 2007; 173:605-616.

15. Minka NS, Ayo JO. Physiological responses of food animals to road transportation stress. Afr J Biotechnol 2009; 8(25):7415-7427.

16. Mormède $\mathrm{P}$, Andanson $\mathrm{S}$, Aupérin $\mathrm{B}$, Beerda B. Guémené $D$, Malmkvist $J$, et al. Exploration of the hypothalamic-pituitaryadrenal function as a tool to evaluate animal welfare. Physiol Behav 2007; 92:317-339.

17. Trevisi E, Bertoni G. Some physiological and biochemical methods for acute and chronic stress evaluation in dairy cows. Ital J Anim Sci 2009; 8(Supp. 1):265-286.

18. Herskin MS, Munksgaard L, Ladewig J. Relations between adrenocortical and nociceptive responses toward acute stress in individual dairy cows. Physiol Behav 2004; 83(3):411-420. 
19. Amtmann VA, Gallo C, van Schaik G, Tadich N. Relaciones entre el manejo ante-mortem, variables sanguíneas indicadoras de estrés y $\mathrm{pH}$ de la canal en novillos. Arch Med Vet 2006; 38(3):259-264.

20. Knowles T, Warriss P. Stress physiology of animals during transport. In: Livestock handling and transport, CABI: 2006.

21. Mounier L, Dubroeucq $\mathrm{H}$, Andanson $\mathrm{S}$, Veissier I. Variations in meat $\mathrm{pH}$ of beef bulls in relation to conditions of transfer to slaughter and previous history of the animals. J Anim Sci 2006; 84:1567-1576.

22. Cooke RF, Bohnert DW. Technical note: Bovine acute-phase response after corticotrophinrelease hormone challenge. J Anim Sci 2011; 89:252-257.

23. Schwartzkopf-Genswein KS, Haley DB, Church S, Woods J, O 'byrne T. An education and training programme for livestock transporters in Canada. Vet Ital 2008; 44(1):273-283.

24. Gallo C. Transporte e bem-estar animal. Ciênc vet tróp 2008; 11 Suppl 1:70-79.

25. Gallo C, Lizondo G, Knowles G. Effects of journey and lairage time on steers transported to slaughter in Chile. Vet Rec 2003; 152:361-364.

26. Miranda-de la Lama GC, Rivero L, Chacón G, Garcia-Balenguer S, Villarroel M, Maria GA. Effect of the pre-slaughter logistic chain on some indicators of welfare in lambs. Livest Sci 2010; 128:52-59.

27. Gavinelli A, Ferrara M, Simonin D. Formulating policies for the welfare of animals during long distance transportation. Vet Ital 2008; 44(1):71-86.

28. Resolución 002341/2007, (Boletín del Estado, número 46730, de 24-09-07) Bogotá: Instituto Colombiano Agropecuario ICA: 2007.

29. Ljungberg D, Gebresenbet G, Aradom S. Logistics chain of animal transport and abattoir operations. Biosystems Eng 2007; 96(2):267-277.

30. Immonen $\mathrm{K}$, Puolanne $\mathrm{E}$. Variation of residual glycogen-glucose concentration al ultímate $\mathrm{pH}$ values below 5.75. Meat Sci 2000; 55:279-283.
31. Muchenje V, Dzama K, Chimonyo M, Strydom PE, Hugo A, Raats JG. Some biochemical aspects pertaining to beef eating quality and consumer health: A review. Food Chem 2009; 112:279-289.

32. Edge MK, Barnett JL. Development of animal welfare standards for the livestock transport industry: process, challenges, and implementation. J Vet Behav 2009; 4:187-192.

33. Hambrecht E, Eissen JJ, Newman DJ, Smith $\mathrm{CH}$, Versteen MW, Den Hartog LA. Preslaughter handling effects on pork quality and glycolytic potential in two muscles differing in fiber type composition. J Anim Sci 2005; 83:900-907.

34. Mach N, Bach A, Velarde A, Devant $M$. Association between animal, transportation, slauthterhouse practices, and meat $\mathrm{pH}$ in beef. Meat Sci 2008; 78:232-238.

35. King DA, Schuehle CE, Randel RD, Welsh TH, Oliphint RA, Baird BE, et al. Influence of animal temperament and stress responsiveness on the carcass quality and beef tenderness of feedlot cattle. Meat Sci 2006; 74:546-556.

36. Jeleníková J, Pipek P, Staruch L. The influence of ante-mortem treatment on relationship between $\mathrm{pH}$ and tenderness of beef. Meat Sci 2008; 80:870-874.

37. Decreto 3149/2006, (Boletín del Estado, número 46390, de 13-09-06) Bogotá: Ministerio de Agricultura y Desarrollo Rural; 2006.

38. Decreto 414/2007, (Boletín del Estado, número 46543, de 15-02-07). Bogotá: Ministerio de Agricultura y Desarrollo Rural; 2007.

39. Decreto 1500/2007, (Boletín del Estado, número 46623, de 04-05-07). Bogotá: Ministerio de la Protección Social; 2007.

40. Resolución 2905/2007, (Boletín del Estado, número 46733, de 27-08-07). Bogotá: Ministerio de la Protección Social; 2007.

41. Terlow EMC, Arnould D, Auperin B, Berri C, Bihan-Duval E, Deiss $V$ et al. Pre-slaughter conditions, animal stress and welfare: current status and possible future research. Animal 2008; 2(10):1501-1517.

42. Blokhuis HJ, Keeling LJ, Gavinelli A, Serratosa J. Animal welfare's impact on the food chain. Trends Food Sci Technol 2008; 19:579-587. 